\title{
Absolute stability and dynamical stabilisation in predator-prey systems
}

\section{Ayawoa S. Dagbovie \& Jonathan A. Sherratt}

Journal of Mathematical Biology

ISSN 0303-6812

Volume 68

Number 6

J. Math. Biol. (2014) 68:1403-1421

DOI 10.1007/s00285-013-0672-8

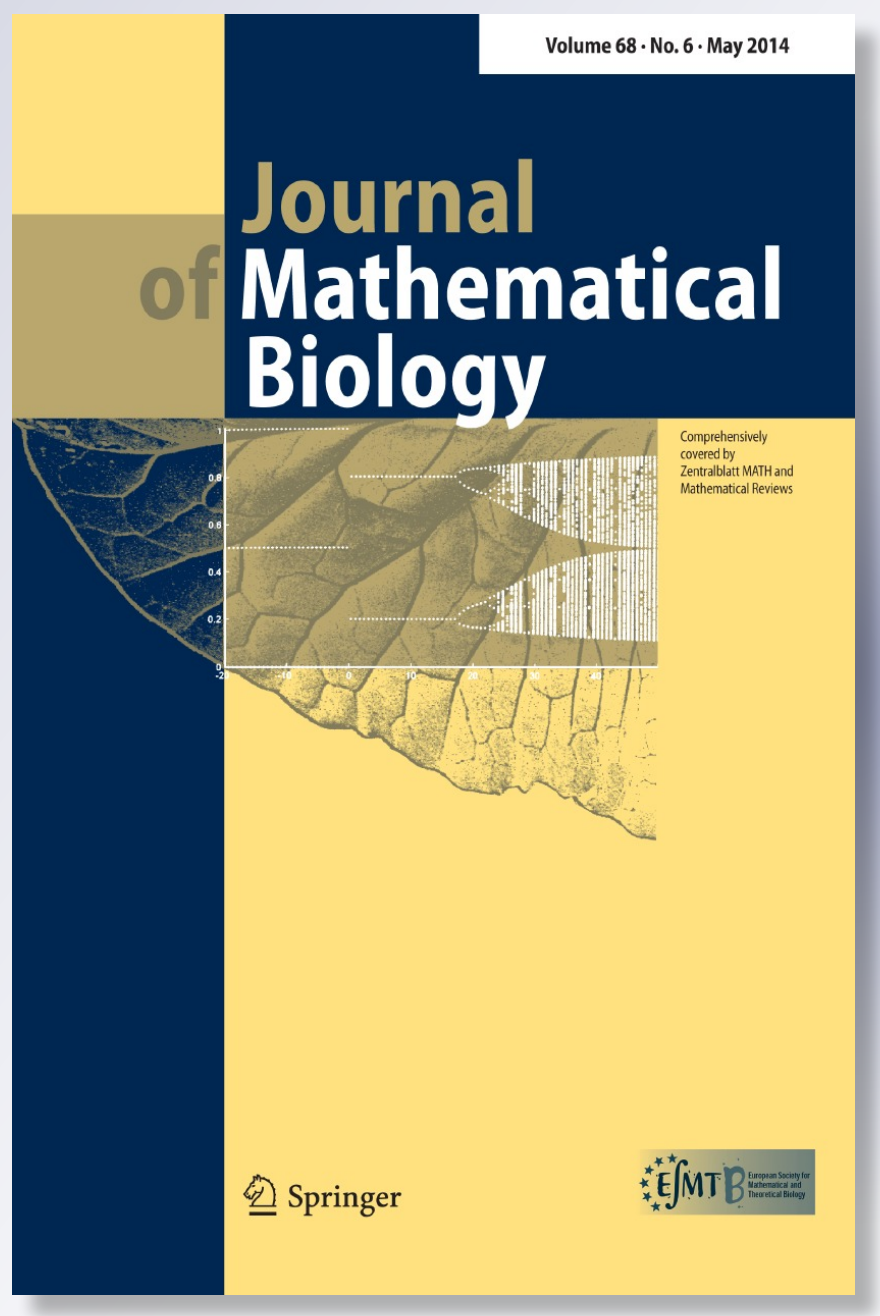

Springer 
Your article is protected by copyright and all rights are held exclusively by SpringerVerlag Berlin Heidelberg. This e-offprint is for personal use only and shall not be selfarchived in electronic repositories. If you wish to self-archive your article, please use the accepted manuscript version for posting on your own website. You may further deposit the accepted manuscript version in any repository, provided it is only made publicly available 12 months after official publication or later and provided acknowledgement is given to the original source of publication and a link is inserted to the published article on Springer's website. The link must be accompanied by the following text: "The final publication is available at link.springer.com". 


\title{
Absolute stability and dynamical stabilisation in predator-prey systems
}

\author{
Ayawoa S. Dagbovie · Jonathan A. Sherratt
}

Received: 21 October 2012 / Revised: 8 March 2013 / Published online: 10 April 2013

(C) Springer-Verlag Berlin Heidelberg 2013

\begin{abstract}
Many ecological systems exhibit multi-year cycles. In such systems, invasions have a complicated spatiotemporal structure. In particular, it is common for unstable steady states to exist as long-term transients behind the invasion front, a phenomenon known as dynamical stabilisation. We combine absolute stability theory and computation to predict how the width of the stabilised region depends on parameter values. We develop our calculations in the context of a model for a cyclic predator-prey system, in which the invasion front and spatiotemporal oscillations of predators and prey are separated by a region in which the coexistence steady state is dynamically stabilised.
\end{abstract}

Keywords Ecological invasion · Absolute stability $\cdot$ Vole $\cdot$ Population cycles

Mathematics Subject Classification (2000) $\quad 92 \mathrm{D} 40 \cdot 35 \mathrm{C} 07 \cdot 35 \mathrm{~K} 57$

\section{Introduction}

Ecological invasion is a widespread phenomenon with important environmental and economic impacts (Matsumura et al. 2004; Tokarska-Guzik et al. 2008). Moreover, invasions are occurring with increasing frequency due to climate change, which can enable previously non-invasive species to invade (Hellmann et al. 2008). This paper

\footnotetext{
A. S. Dagbovie $(\varangle) \cdot$ J. A. Sherratt

Department of Mathematics and Maxwell Institute for Mathematical Sciences,

Heriot-Watt University, Edinburgh EH14 4AS, UK

e-mail: ad195@hw.ac.uk

J. A. Sherratt

e-mail: jas@ma.hw.ac.uk
} 
concerns one particular class of invasions: the spread of a predator into a population of its prey. This process has been explored in many mathematical modelling studies using models formulated as partial differential equations (pdes), integrodifference equations, cellular automata and others (Dunbar 1984; Sherratt et al. 2000; Owen and Lewis 2001; Sherratt et al. 1997; Sherratt 2001). In simple cases, invasion takes the form of a transition wavefront, with a prey-only equilibrium ahead of the front and constant coexistence of predator and prey behind it. In such cases, the main question of interest is the dependence of the invasion speed on ecological parameters.

A characteristic feature of predator-prey interactions is their ability to generate population cycles. This feature of predator-prey models dates back to the original work of Lokta and Volterra (Lotka 1925; May and Mclean 2007; Turchin 2003; Volterra 1926), and empirical studies have confirmed predation as the cause of cycling in some prey populations (Korpimäki et al. 2002). For cyclic interactions, invasion of prey by predators is inevitably more complicated: the constant coexistence of predators and prey is unstable; therefore homogeneity cannot be expected as the only behaviour behind the invasion front. Instead, a number of modelling studies have predicted spatiotemporal oscillations organised into periodic travelling waves (Garvie 2007; Petrovskii et al. 2000; Sherratt 2001). Such waves have been found in spatiotemporal data sets on a number of natural populations (Sherratt and Smith 2008), with invasion being among the factors proposed for their generation.

The change from a simple invasive transition wave to a periodic wave behind the invasion front as parameters are varied occurs because the coexistence equilibrium becomes unstable. However, numerical simulations show that, in many cases, the invasion front and the periodic travelling wave are separated by a region in which the solution is approximately at the coexistence equilibrium. In the early stages of an invasion, this region expands until it eventually reaches a maximum width which then remains constant as the invasion proceeds (see Fig. 1 for a typical space-time plot showing the dependence on time of the width). The existence of a region in which the solution is approximately at the unstable coexistence steady state is known as "dynamical stabilisation" (Malchow and Petrovskii 2002; Malchow et al. 2008; Petrovskii and Malchow 1998; Petrovskii et al. 2001) and it is a major feature of predator-prey invasions for a wide range of parameter values.

Although the analysis in this paper is quite general, all of our numerical examples are for the Rosenzweig-MacArthur (1963) model which assumes logistic growth for the prey population and a Holling type 2 response to predation:

$$
\begin{gathered}
u_{t}=D_{u} u_{x x}+f(u, v) \\
v_{t}=D_{v} v_{x x}+g(u, v) \\
\text { with } f(u, v)=u(1-u)-\frac{k u v}{1+k u} \\
g(u, v)=\frac{1}{b}\left(\frac{k u v}{1+k u}\right)-\frac{v}{a b} .
\end{gathered}
$$

Here the variables $u$ and $v$ are prey and predator densities at time $t$ and spatial location $x$ in a one dimensional domain. We will focus on two different values of $b$; firstly 


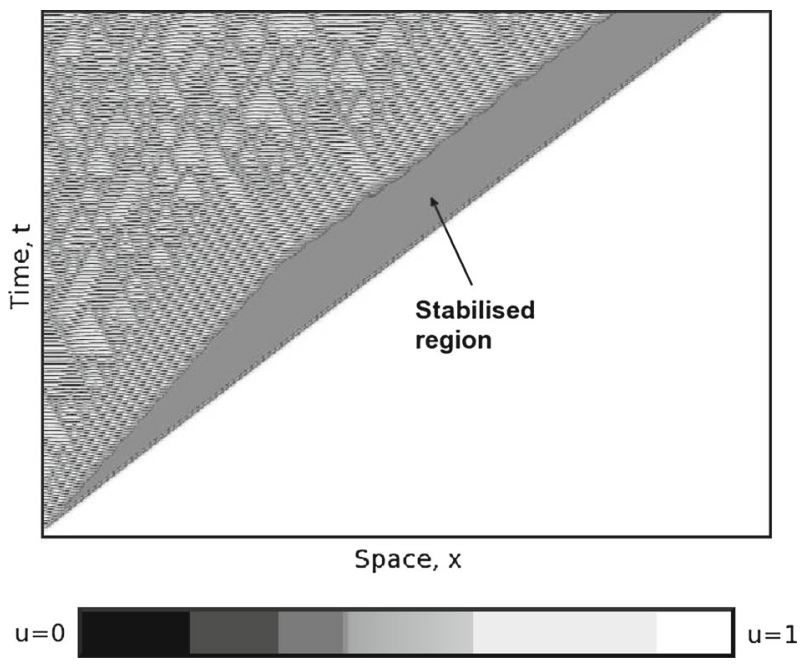

Fig. 1 Space-time plot showing how the edges of the stabilised region vary with time for $b=3.0$ and $k=8.2$. All other parameters are as in (1e). The space variable $x$ varies between 0 and 4,000, with $t$ between 0 and 5,660. As $t$ increases from zero, the stabilised region expands until $t \simeq 2745$, after which the width remains constant. We have solved (1) numerically using a semi-implicit finite difference method with a grid spacing of 0.5 and time step of $10^{-2}$. Our initial condition is given by the prey-only state $u(x, 0)=1$ and $v(x, 0)=0$ with a slight perturbation near $x=0$; we assume that there is no flux on both ends of the domain giving the boundary conditions $u_{x}=v_{x}=0$ at $x=0$ and $x=4000$. Note that the scale bar has been deliberately made discrete, in order to achieve better clarity

$b=3.0$ and later $b=1.2$. In both cases, we vary $k$ with the other parameter values fixed as follows:

$$
a=1.3, D_{u}=1 \text { and } D_{v}=2 \text {. }
$$

The model $(1 \mathrm{a}-\mathrm{d})$ has a unique coexistence steady state $\left(u^{*}, v^{*}\right)$ with $u^{*}=1 /(a k-k)$ and $v^{*}=\left(1-u^{*}\right)\left(1+k u^{*}\right) / k$. Standard linear stability analysis indicates that $\left(u^{*}, v^{*}\right)$ is unstable for $k \geq(a+1) /(a-1)=k_{\min }$ and stable to homogeneous perturbations for $k<k_{\min }$.

Figure 2 shows numerical simulations of invasions using (1), for four different values of $k$, with the other parameters fixed (as in (1e), with $b=3.0$ ). In $(a)$, $k<k_{\min }=7.67$ and the system settles to the stable coexistence steady state $\left(u^{*}, v^{*}\right)$ behind the invasion front. In $(b)$ and $(c)$ there is a clear "stabilised region" in which the solution is very close to $\left(u^{*}, v^{*}\right)$, before the onset of spatiotemporal oscillations. Note that the stabilised region is wider for $(b)$ which has the smaller of the two $k$ values. In $(d)$, there is no dynamical stabilisation, and spatiotemporal oscillations develop immediately behind the invasion front.

The phenomenon of dynamical stabilisation has been studied in detail by Petrovskii et al. (2000, 2001); Malchow and Petrovskii (2002). Their numerical simulations were run for relatively short times, and as a result they only observed the initial phase of the solution, in which the width of the stabilised region grows at a constant rate. Petrovskii et al. (2001); Malchow and Petrovskii (2002) calculated the growth rate by 

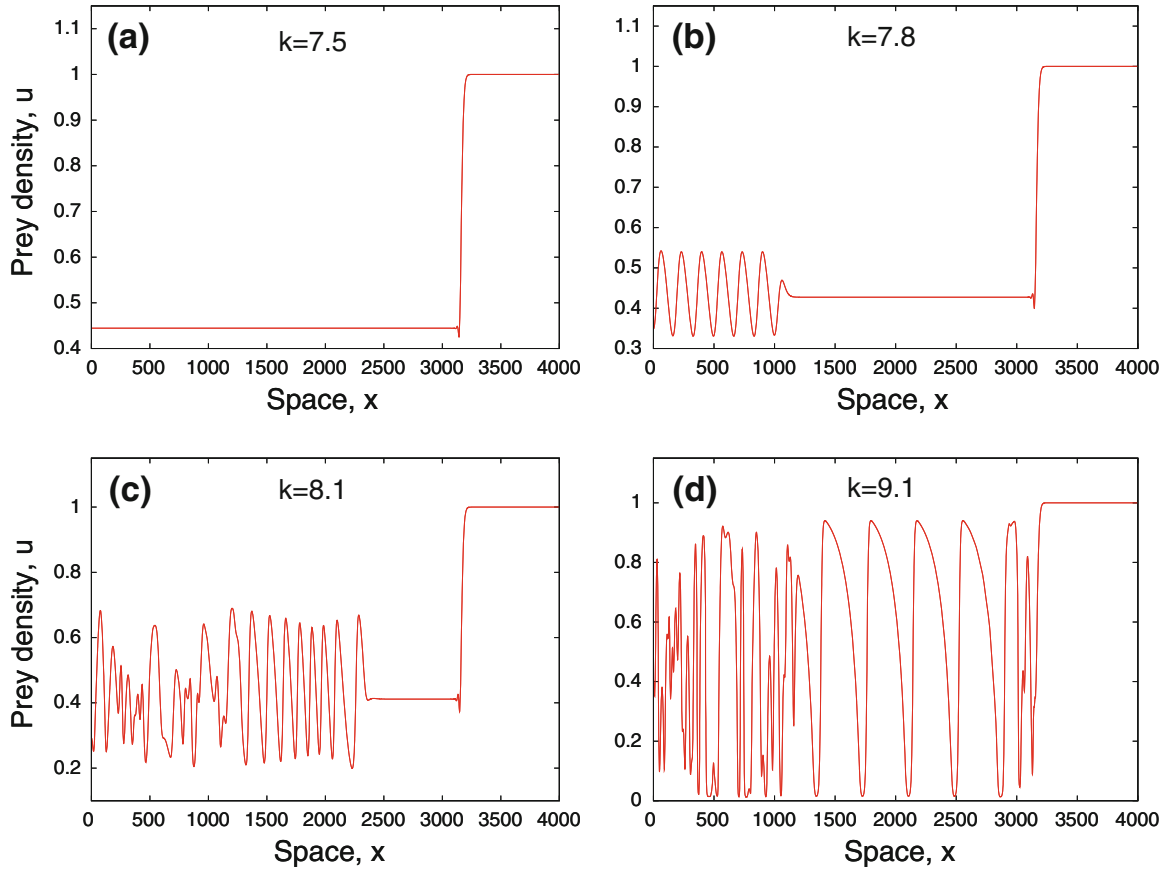

Fig. 2 Numerical simulations of invasion in (1) for four different values of $k$. Here $b=3.0$ and the other parameters are given in (1e). At time $t=0$, the unstable steady state $(1,0)$ is slightly perturbed near $x=0$. a Illustrates the structure of a stable solution: it simply consists of an invasion front followed by the coexistence steady state. b-c Show a prey-only region followed by an invasion front, itself followed by a stabilised region which then gives way to oscillations. These plots also suggest that the width of the stabilised region decreases as $k$ increases. For bigger values of $k$, that region is absent as we can observe in (d). In each case, $x \in(0,4000)$ and the details of the numerical method were as in Fig. 1

applying linear spreading speed theory (van Saarloos 2003) to the interface between the coexistence steady state and spatiotemporal oscillations. The condition for dynamical stabilisation to occur at all is that this interface moves more slowly than the invasion front. Similar calculations were done by Nozaki and Bekki (1983) in their study of periodic wave generation in the complex Ginzburg-Landau equation.

The objective of this paper is an improved mathematical understanding of dynamical stabilisation. In contrast to Petrovskii et al. (2001); Malchow and Petrovskii (2002), our focus is on the width of the stabilised region once it has become constant, after the initial growth phase. In particular, we present a method that enables calculation of the dependence on parameter values of the width of the stabilised region. Our approach is based on the theory of absolute stability. In Sect. 2, we discuss the notions of absolute and convective stability for spatiotemporal systems, which will be our key tools for determining the extent of dynamical stabilisation in Sects. 3 and 4.

\section{Convective and absolute stability}

The phenomenon of "dynamical stabilisation" illustrated in Fig. 2b, c is an intuitively surprising one: the coexistence steady state is unstable even to spatially homogeneous 
perturbations and yet it appears as a long term feature of the solution. An understanding of this behaviour requires the theory of convective and absolute stability, which we now summarise. In temporal systems, if a solution is locally stable any small perturbation decays over time. However, in a spatiotemporal context one must consider also the propagation of growing perturbations. A solution is "convectively unstable" if all unstable linear modes propagate as they grow. By contrast, "absolutely unstable" solutions have stationary unstable linear modes. A more detailed discussion of these concepts is given in Sandstede and Scheel (2000).

Our investigation of dynamical stabilisation is based on the absolute stability of the coexistence steady state in a frame of reference moving with an arbitrary velocity $V$. Therefore we rewrite (1) as

$$
\begin{gathered}
u_{t}=D_{u} u_{z z}+V u_{z}+f(u, v) \\
v_{t}=D_{v} v_{z z}+V v_{z}+g(u, v)
\end{gathered}
$$

where $z=x-V t$.

We now summarise a numerical method for calculating whether the coexistence equilibrium $\left(u^{*}, v^{*}\right)$ of (2) is absolutely stable; the method is discussed at greater length by Rademacher et al. (2007). The first step is to compute the equations satisfied by small perturbations to the steady state $\left(u^{*}, v^{*}\right)$. We obtain these by linearising (2) about $\left(u^{*}, v^{*}\right)$. We look for solutions of the form

$$
(u, v)=\left(u^{*}, v^{*}\right)+(\bar{u}, \bar{v}) e^{\lambda t+v z}
$$

where $\lambda$ is the temporal eigenvalue and $v$ is the corresponding spatial eigenvalue $(\lambda, v \in \mathbb{C}) ; \bar{u}$ and $\bar{v}$ are complex valued constants. Substituting (3) into (2) and neglecting terms that are nonlinear in $\bar{u}$ and $\bar{v}$ gives

$$
A(\lambda, v) y=0
$$

where

$$
\begin{aligned}
A(\lambda, v) & =\left(\begin{array}{cc}
D_{u} v^{2}+V v+\left.\frac{\partial f}{\partial u}\right|_{\left(u^{*}, v^{*}\right)}-\lambda & \left.\frac{\partial f}{\partial v}\right|_{\left(u^{*}, v^{*}\right)} \\
\left.\frac{\partial g}{\partial u}\right|_{\left(u^{*}, v^{*}\right)} & D_{v} v^{2}+V v+\left.\frac{\partial g}{\partial v}\right|_{\left(u^{*}, v^{*}\right)}-\lambda
\end{array}\right) \\
\text { and } y & =\left(\begin{array}{c}
\bar{u} \\
\bar{v}
\end{array}\right)
\end{aligned}
$$

The dispersion relation $\mathcal{D}(\lambda, v)=\operatorname{det}[A(\lambda, v)]$ must be zero for non-trivial solutions. For fixed $\lambda, \mathcal{D}$ is a fourth order polynomial in $v$ and we denote its four roots by $\nu_{1}(\lambda)$, $v_{2}(\lambda), v_{3}(\lambda)$ and $v_{4}(\lambda)$, repeated with multiplicity and indexed such that

$$
\operatorname{Re}\left(v_{1}\right) \geq \operatorname{Re}\left(v_{2}\right) \geq \operatorname{Re}\left(v_{3}\right) \geq \operatorname{Re}\left(v_{4}\right)
$$


Then the "absolute spectrum" of $\left(u^{*}, v^{*}\right)$ is $\Sigma_{a b s}=\left\{\lambda \mid \operatorname{Re}\left[v_{2}(\lambda)\right]=\operatorname{Re}\left[v_{3}(\lambda)\right]\right\}$, and $\left(u^{*}, v^{*}\right)$ is absolutely stable if and only if $\Sigma_{a b s}$ only contains eigenvalues with negative real part (Sandstede and Scheel 2000).

The larger set $\left\{\lambda \mid \operatorname{Re}\left[v_{i}(\lambda)\right]=\operatorname{Re}\left[v_{j}(\lambda)\right]\right.$, for some $\left.i \neq j\right\}$ is known as the "generalised absolute spectrum" (Rademacher et al. 2007). We calculate the absolute spectrum using the method of Rademacher et al. (2007) which involves first calculating the entire generalised absolute spectrum and then determining which parts of it form the absolute spectrum.

Calculation of the Generalised Absolute Spectrum To calculate the generalised absolute spectrum, we first compute the pairs $\left(\lambda_{*}, v_{*}\right)$ that solve

$$
\begin{aligned}
\mathcal{D}(\lambda, \nu) & =0 \\
\partial_{\nu} \mathcal{D}(\lambda, \nu) & =0 ;
\end{aligned}
$$

these are known as branch points. Note that $\nu_{*}$ is a repeated root of $\mathcal{D}\left(\lambda_{*}, v\right)=0$ and therefore any branch point is in the generalised absolute spectrum. The approach in Rademacher et al. (2007) is to calculate the generalised absolute spectrum by continuing it numerically using the branch points as starting points. General theory (Rademacher et al. 2007) implies that the generalised absolute spectrum is a union of connected components, each of which emanates from a branch point. Therefore this numerical continuation procedure calculates the whole generalised absolute spectrum, and the part of this that is absolute spectrum can be determined by monitoring all four roots for $v$ at each point. Full details of this numerical approach and its practical implementation are given in Rademacher et al. (2007), Smith et al. (2009).

Figure 3 shows two examples of generalised absolute spectra, calculated in this way. A key feature of these plots is that the most unstable points of the absolute spectrum are branch points. This property has been proved for some simpler equations (Smith et al. 2009), although it does not hold in general: there are some equations for which the absolute spectrum extends to the right of the most unstable branch point (Rademacher et al. 2007). Our study of dynamical stabilisation will depend fundamentally on the most unstable point in the absolute spectrum. Therefore we calculated the absolute spectrum of (2) for a range of values of $k$ and $V$. In every case, the most unstable points in the absolute spectrum were branch points, and in our subsequent calculations we will assume that this property holds. Calculations of the most unstable points in the absolute spectrum then reduces to a straightforward solution of polynomials. Elimination of $\lambda$ from (5a) and (5b) gives a sixth order polynomial in $\nu$. For each of the six roots, we calculate the corresponding $\lambda$. We then substitute this $\lambda$ value back into $\mathcal{D}(\lambda, v)=0$, giving a quartic polynomial for $\nu$. Two of the roots of this quartic will be the repeated root for $v$ that has already been calculated; a comparison of the real parts of this repeated root and the real parts of the other two roots enables us to determine whether or not the repeated roots are $v_{2}$ and $v_{3}$, which is the condition for the branch point to be in the absolute spectrum. 

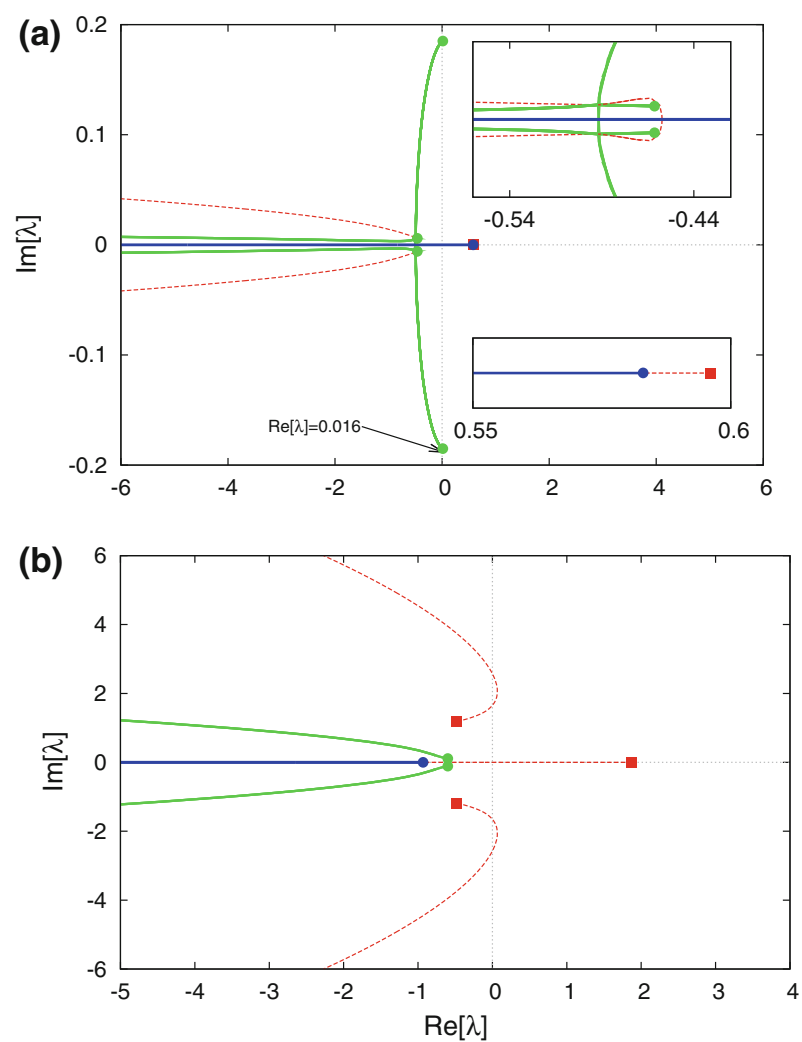

Fig. 3 Plots of the generalised absolute spectrum for $b=3, k=9$ and two different values of the velocity $V$. The green solid line is the absolute spectrum and small green disks represent its branch points. The absolute spectrum contains all temporal eigenvalues such that the corresponding spatial eigenvalues satisfy $\operatorname{Re}\left[v_{2}\right]=\operatorname{Re}\left[v_{3}\right]$. We show as a red dashed line those $\lambda$ 's characterised by $\operatorname{Re}\left[v_{1}(\lambda)\right]=\operatorname{Re}\left[v_{2}(\lambda)\right]$; branch points of this set are shown as red filled squares. In both cases, the right-most points in the absolute spectrum are branch points. The blue solid line and blue disks are the remainder of the generalised absolute spectrum and its branch points, for which $\operatorname{Re}\left[v_{3}(\lambda)\right]=\operatorname{Re}\left[v_{4}(\lambda)\right]$. The top panel corresponds to $V=0.01$ and the bottom one corresponds to $V=2$. Note that for $V=0.01$, four of the six branch points belong to the absolute spectrum, and the most unstable of these (a complex conjugate pair) are in the right half plane: the steady state is absolutely unstable. In the $V=2$ case however, only two of the branch points belong to the absolute spectrum and they are in the left half plane: the solution is absolutely stable. The inserts show details of behaviour near branch points for $V=0.01$; the range on the vertical axis is $-0.035<\operatorname{Im}(\lambda)<0.035$ for the top insert and $-1<\operatorname{Im}(\lambda)<1$ for the bottom insert (colour figure online)

\section{Calculating the extent of dynamical stabilisation}

We denote by $\lambda_{\max }$ the most unstable point in the absolute spectrum of $\left(u^{*}, v^{*}\right)$. Figure 4 illustrates $\operatorname{Re}\left[\lambda_{\max }\right]$ as a function of the reference frame velocity $V$ for one value of $k$; the qualitative form remains the same as $k$ is varied. In particular, $\operatorname{Re}\left[\lambda_{\max }\right]>0$ on an interval of $V$ values, say $-V^{*}<V<V^{*}$, with $\operatorname{Re}\left[\lambda_{\max }\right]<0$ otherwise. Note that $\operatorname{Re}\left[\lambda_{\max }\right]$ is symmetric about $V=0$ : this is due to the directional symmetry of (1). The plot of $\operatorname{Re}\left[\lambda_{\max }\right]$ against $V$ provides the basis for our calculation 


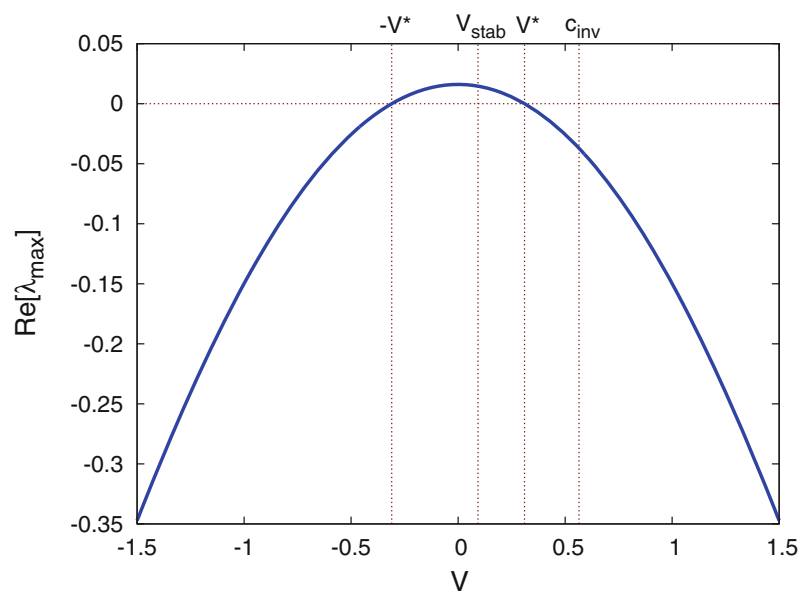

Fig. 4 Plot of $\operatorname{Re}\left[\lambda_{\max }(V)\right]$, the maximum growth rate of perturbations to the steady state in a frame of reference moving with velocity $V$, for $b=3.0, k=8.0$. Here $c_{i n v}>V^{*}$ which means that the invasion front is moving faster than all growing linear modes so that dynamical stabilisation will occur for these parameter values

of the width of the stabilised region. Our arguments follow directly those used to calculate the width of wavetrain bands in Sherratt et al. (2009), Smith and Sherratt (2009), and the description below is deliberately brief. The coexistence steady state that develops immediately behind the invasion front will be perturbed by the front. We make the reasonable assumption that this perturbation contains all unstable linear modes. To understand the subsequent behaviour of these perturbations, we will visualise the dynamics in the space-time plane (Fig. 5). We denote by $\left(x^{*}, t^{*}\right)$ a point on the invasion front. When $t$ increases above $t^{*}$, the perturbations applied to the steady state $\left(u^{*}, v^{*}\right)$ by the invasion front spread out in time and space, growing along all rays $x=x^{*}+$ $\left(t-t^{*}\right) V$ with $V$ between $-V^{*}$ and $V^{*}$. In order to calculate precisely the width of the stabilised region, we must give a precise definition of its ends. The right-hand end is simply the invasion front, and we define the left-hand end to be the point at which perturbations applied to the steady state $\left(u^{*}, v^{*}\right)$ by the invasion front first become amplified by a factor $\mathcal{F}$. This occurs at time $t_{\text {crit }}(V)=t^{*}+\log (\mathcal{F}) / \operatorname{Re}\left[\lambda_{\max }(V)\right]$, with the corresponding location being $x_{\text {crit }}(V)=x^{*}+V \log (\mathcal{F}) / \operatorname{Re}\left[\lambda_{\max }(V)\right]$. Note that $\mathcal{F}>0$ can be chosen arbitrarily, but we will show that the parameter dependence of the width of the stabilised region is independent of the choice of $\mathcal{F}$. Figure 5 shows an example of the curve $\left(x_{\text {crit }}(V), t_{c r i t}(V)\right),\left(-V^{*}<V<V^{*}\right)$. The left-hand edge of the stabilised region occurs at the point on this curve that is closest to the invasion front $x=x^{*}+\left(t-t^{*}\right) c_{i n v}$, where $c_{i n v}$ is the invasion speed.

A straightforward calculation shows that this closest approach occurs when $V$ satisfies

$$
\left(V-c_{i n v}\right) \operatorname{Re}\left[v_{\max }(V)\right]=\operatorname{Re}\left[\lambda_{\max }(V)\right], \quad-V^{*}<V<V^{*} .
$$




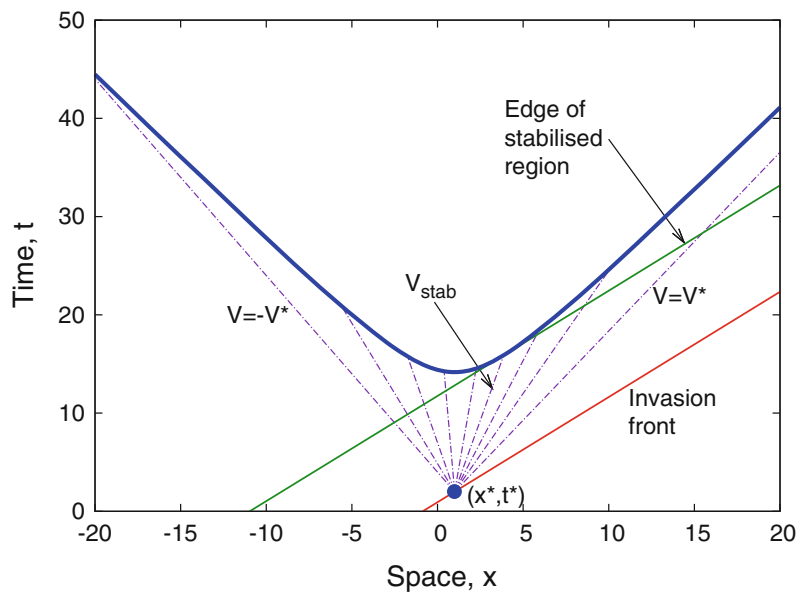

Fig. 5 An illustration of the argument on which our calculation of the width of the stabilised region is based. The thick blue curve is a plot of calculated values, not a sketch, and the parameters are given by (1e) with $b=1.2, k=9.0$ and $\mathcal{F}=2$; this value of $\mathcal{F}$ is very much smaller than any suitable real value, but is useful for illustration purposes. Then, if we view the point $\left(x^{*}, t^{*}\right)=(1,2)$ in a frame of reference moving at a velocity $V$, with $-V^{*}<V<V^{*}$, perturbations grow and spread out in time and space, reaching the point $\left(x_{\text {crit }}, t_{\text {crit }}\right)$ (blue curve) when their amplitude has doubled. Perturbations moving at the velocity $V_{\text {stab }}$ reach the edge of stabilised region closest to the invasion front (colour figure online)

Numerical calculations indicate that (6) has a unique solution for $V$, which we denote by $V_{\text {stab }}$. Figure 8 shows a plot of $V_{\text {stab }}$ and $V^{*}$ as functions of $k$. The steady state $\left(u^{*}, v^{*}\right)$ changes stability at $k_{\text {min }} \simeq 7.67$ and below this value, $V^{*}$ and $V_{\text {stab }}$ are not defined. As $k$ increases from 7.67, $V^{*}$ and $V_{\text {stab }}$ both increase from zero. Initially $V^{*}>$ $V_{\text {stab }}>0$ and at $k_{\max }=9.027, V^{*}=V_{\text {stab }}=c_{\text {inv }}$. For $k>9.027$, perturbations are able to outrun the invasion front, and there is no stabilised region. Note that the condition $V^{*}>V_{\text {stab }}$ for dynamical stabilisation to occur matches the condition of Petrovskii et al. (2001), which is based on linear spreading speeds, because of the known relationship between spreading speeds and absolute stability (van Saarloos 2003). For $7.67<k<9.027$, perturbations travel more slowly than the invasion and dynamical stabilisation occurs (Fig. 6).

The width of the stabilised region is the spatial distance between the invasion front and $\left(x_{\text {crit }}\left(V_{\text {stab }}\right), t_{\text {crit }}\left(V_{\text {stab }}\right)\right)$, which can be simplified to

$$
\mathcal{L}(k)=\frac{-1}{\operatorname{Re}\left[v_{\max }\left(V_{\text {stab }}\right)\right]} \log (\mathcal{F})
$$

(see Sherratt et al. 2009 for more details). Of course, this width depends on the arbitrary factor $\mathcal{F}$, but crucially all of the parameter dependence decouples from $\mathcal{F}$ and appears only in the coefficient

$$
\mathcal{W}(k)=\frac{-1}{\operatorname{Re}\left[v_{\max }\left(V_{\text {stab }}\right)\right]} .
$$




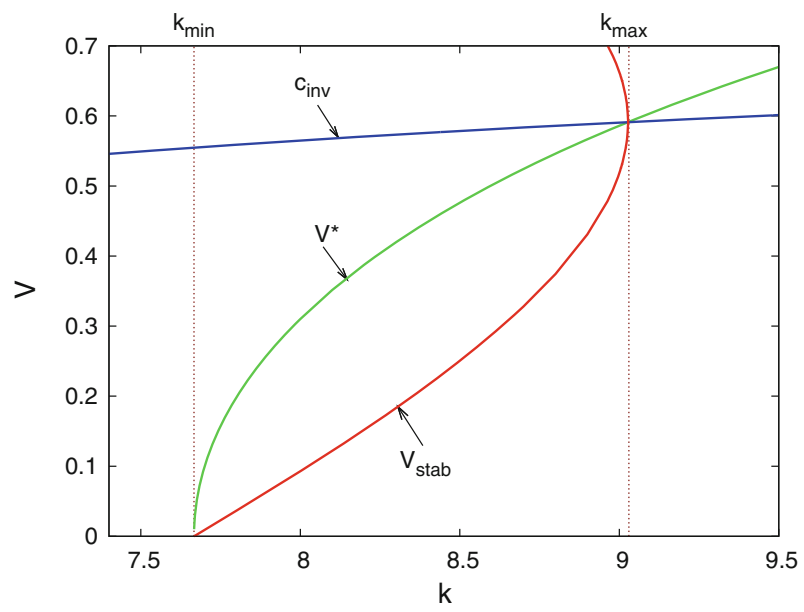

Fig. 6 Plots of $V^{*}, V_{\text {stab }}$ and $c_{i n v}$ against $k$ for $b=3.0 ; a, D_{u}, D_{v}$ are given by (1e). At $k=k_{\text {min }}$, the coexistence steady state becomes unstable. This value of $k$ is also characterised by $V^{*}=V_{\text {stab }}=0$. $V^{*}$ and $V_{\text {stab }}$ increase with $k$ until $k=k_{\max }$. For $k \in\left(k_{\min }, k_{\max }\right)$, we have $V_{\text {stab }}<V^{*}<c_{i n v}$, so that the unstable steady state is dynamically stabilised for these $k$ values. The $V_{\text {stab }}$ curve folds at $k=k_{\max }$ while intersecting both the $V^{*}$ and $c_{i n v}$ curves. For $k>k_{\max }$, the velocity $V_{\text {stab }}$ does not exist as Eq. (6) has no solution. Meanwhile $V^{*}$ has now become bigger than $c_{i n v}$ : there are unstable linear modes that overtake the invasion and dynamical stabilisation will no longer occur

As a test of our theory, we estimated the width $\mathcal{L}(k)$ of the stabilised region for a number of different values of $k$ in numerical simulations of invasion in (1), and compared these with the calculated values of $\mathcal{W}(k)$. To estimate $\mathcal{L}(k)$ in simulations, we need to determine the left- and right-hand edges of the stabilised region. Keeping in mind that the left-hand end is the first point behind the stabilised region, we define it as the point $x_{1}$ at which $\mathcal{A}=\sqrt{\left(u-u^{*}\right)^{2}+\left(v-v^{*}\right)^{2}}$ equals a small value, taken as 0.005 . We choose the right-hand edge to be the point $x_{2}$ on the invasion front that is halfway between the coexistence steady state and the prey only steady state; that is the point at which $\mathcal{A}=\frac{1}{2} \sqrt{\left(1-u^{*}\right)^{2}+v^{* 2}}$. We estimate both $x_{1}$ and $x_{2}$ by linear interpolation between numerical grid points; of course they are both time-dependent. Now recall that the width of the stabilised region is a function of time and has a growing and then a constant phase. We calculated the average value of $x_{2}-x_{1}$ over the time interval of $(8000,11000)$, which is during the constant phase for all the parameter sets we considered. Numerical details are the same as in Fig. 1 except that we now take a larger domain $(0,8000)$ and that the time variable varies between 0 and 11000 .

Figure 7a plots the value of $\mathcal{L}(k)$, estimated in this way, against calculated values for $\mathcal{W}(k)$. Our theory predicts that these should be linearly related, and this is confirmed by the figure. The slope of the best fit line provides an estimate for $\log (\mathcal{F})$. Note that the best fit line has a small but non-zero (positive) intercept: this corresponds to the edges of the numerically estimated stabilised region being excluded from the theoretical calculations. Combining the best fit line with our formula (8) for $\mathcal{W}(k)$, we can predict actual widths of the stabilised region. Figure $7 \mathrm{~b}$ demonstrates the very 

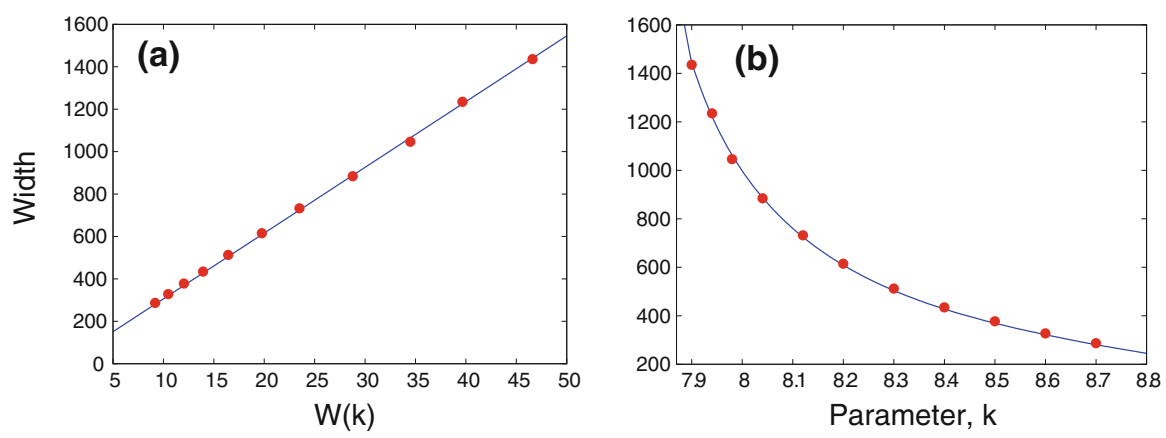

Fig. 7 a Plots the numerically calculated width of the stabilised region $\mathcal{L}(k)$ against $\mathcal{W}(k)$ (red dots); the straight line is the best fit line, given by linear regression. In b we plot our measured width against the parameter $k \in\left[k_{\min }, 8.8\right]$. The blue curve shows the calculated width versus $k$; we obtained the calculated width as a function of $\mathcal{W}(k)$ using the best fit line in (a) (colour figure online)

good comparison between these predictions and the stabilised width measured from simulations, across a wide range of values of $k$.

\section{Point-to-limit cycle invasions}

For smaller values of $b$, the behaviour described in Sect. 3 is augmented by an additional complicating factor. To describe this, we consider in detail the case of $b=1.2$, with $k$ varying and the other parameters given by (1e). Calculations as in Sect. 3 show that $k_{\max }=14.56$ (Fig. 8). However, in numerical simulations, dynamical stabilisation is only observed for $k$ between $k_{\min }=7.67$ and about 10.5 ; above this, spatiotemporal oscillations occur immediately behind the invasion front (illustrated in Fig. 9d). Insight into this is given by comparing space-time plots for values of $k$ above the upper limit for dynamical stabilisation, for $b=3.0$ and $b=1.2$ (Fig. 10). In both cases there are spatiotemporal oscillations immediately behind the invasion front. For $b=3.0$ these are somewhat disordered, and move in the opposite direction to the invasion. However, for $b=1.2$, they have the form of periodic travelling waves moving with the invasion front; further back, there is a transition to more disordered oscillations moving in the opposite direction. Thus for $b=1.2$, the invasion itself consists of a point-to-periodic orbit connection in the travelling wave ODEs, rather than the heteroclinic connection seen for $b=3.0$. When the invasion is of the former type, the solution does not approach the coexistence steady state, and thus dynamical stabilisation of this steady state cannot occur.

General theory (Kopell and Howard 1973) implies that in the travelling wave ODEs corresponding to $(1 \mathrm{a}-\mathrm{d})$, for $k>k_{\min }$, the coexistence steady state undergoes a Hopf bifurcation as the wave speed $c>0$ is increased, at $c=c_{H B}$ say. Calculations of the eigenvalues at this steady state show that they all have negative real parts for $c>c_{H B}$, implying that there cannot be an invasion front of heteroclinic connection type, and numerical simulations indicate that there is instead a point-to-limit cycle invasion front. Note that the existence of this type of front solution of $(1 \mathrm{a}-\mathrm{d})$ has been proved in Dunbar (1986) for $D_{u}=0$ and in Fraile and Sabina (1989) for $D_{u}>0$ sufficiently 


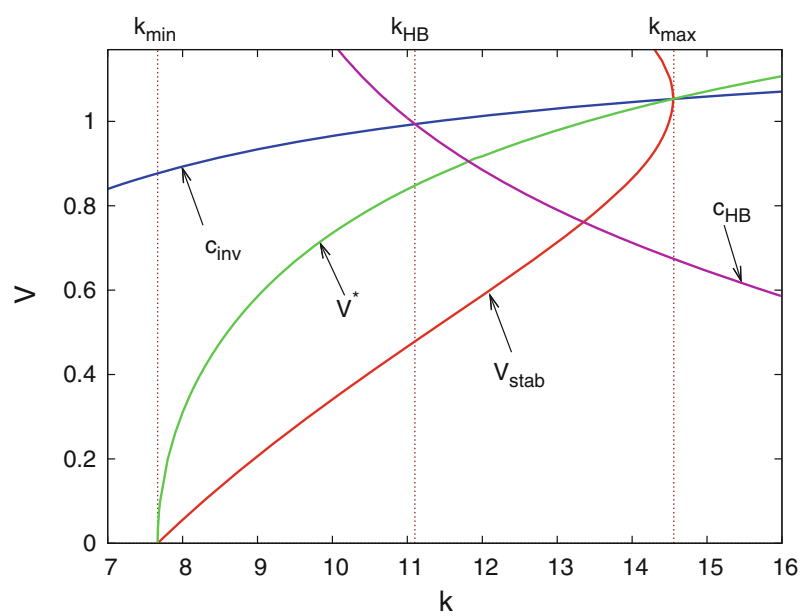

Fig. 8 Plots of $V^{*}, V_{s t a b}, c_{H B}$ and $c_{i n v}$ against $k$ for $b=1.2$. The steady state $\left(u^{*}, v^{*}\right)$ is unstable for values of $k$ above $k_{\min }$. For $k$ in this range, $\left(u^{*}, v^{*}\right)$ has a Hopf bifurcation in the travelling wave equation corresponding to (1) at a critical value of $V$. On this picture, we also show $c_{H B}$, the wave speed at which this Hopf bifurcation occurs, as a function of $k$. Note that the intersection between the $c_{H B}$ and $c_{i n v}$ curves occurs at a value $k_{H B} \simeq 11.1$, which is less than $k_{\max }$
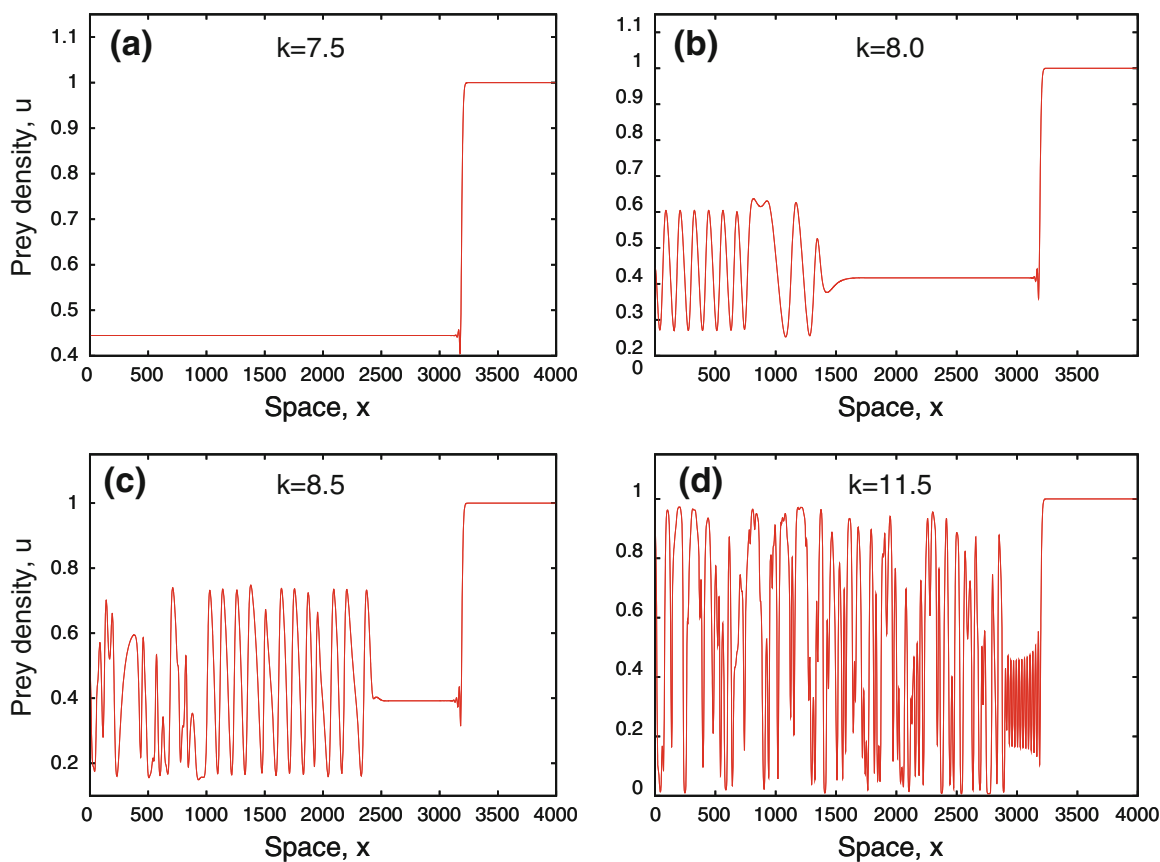

Fig. 9 Numerical simulations of invasion in (1) for $b=1.2$ and four different values of $k$. The other parameters are given by (1e). $\mathbf{d}$ Corresponds to $k=11.5$; even though this value is less than $k_{\max }$, there is no dynamical stabilisation. Initial conditions, boundary conditions and the numerical method were as in Fig. 2 


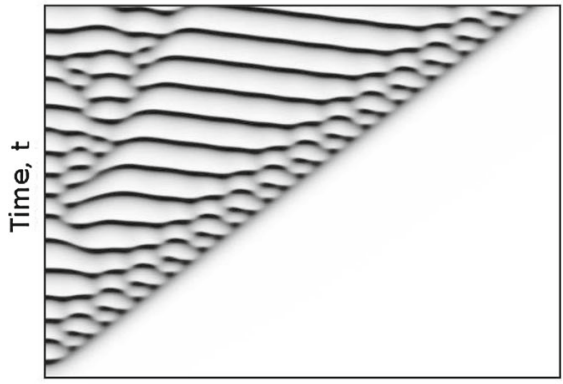

Space, $x$

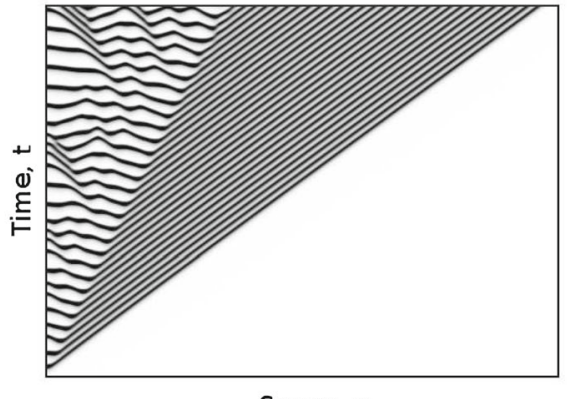

Space, $x$

$$
\mathrm{u}=0
$$
$\mathrm{u}=1$

Fig. 10 Plots of prey densities for $t \in[0,1070]$ with $x \in[0,4000]$. The left panel corresponds to $b=3.0$, $k=9.5$ and shows that the waves behind the invasion front are moving in a direction opposite to that of the front. However in the right panel $(b=1.2, k=15)$ we see the waves initially moving in the same direction as the front; further back there is a transition to waves moving in the opposite direction. Numerical details are the same for Fig. 1, and the other parameter values are given by (1e)
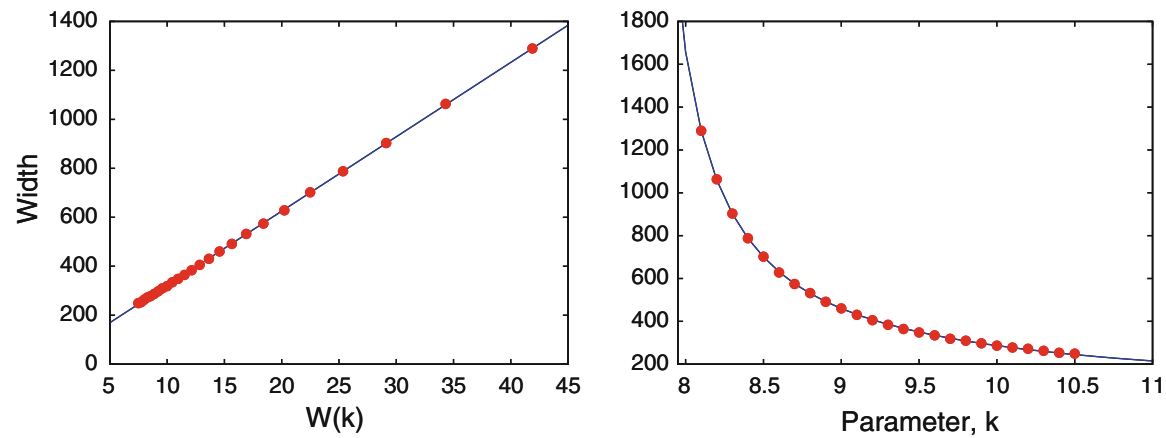

Fig. 11 a The variation in the width $\mathcal{L}(k)$ in numerical simulations of (1) with the width coefficient $\mathcal{W}(k)$ (red dots); the straight line is the best fit line, given by linear regression. In $\mathbf{b}$ we plot our measured band width against the parameter $k \in\left[k_{\min }, 10.5\right]$. The blue curve shows the calculated bandwidth versus $k$ blue (colour figure online)

small. For the case $b=3.0$ considered in Sect. $3, c_{H B}$ is significantly greater than $c_{i n v}$ for all values of $k \in\left[k_{\min }, k_{\max }\right]$, so that this change in the form of the invasion front does not have any effect on dynamical stabilisation. However for $b=1.2, c_{H B}<c_{\text {inv }}$ for $k>11.1$ (see Fig. 8), implying that dynamical stabilisation will only occur for $k_{\text {min }}<k<k_{H B}=11.1$. Here we define by $k_{H B}$ the value of $k$ at which $c_{H B}=c_{i n v}$. In fact, as mentioned earlier, we observe stabilisation only up to about $k=10.5$. A close look at numerical solutions for $k \in(10.5,11.1)$ reveals that the solution behind the invasion front decays relatively slowly and does not get sufficiently close to the coexistence steady state before giving way to the spatiotemporal oscillations. For values of $k$ in the range $k_{\min }<k<10.5$, there is a very close agreement between the predicted width of the stabilised region, calculated as described in Sect. 3, and the width measured in numerical simulations (Fig. 11). 


\section{Extension to other parameter values}

The main results of this paper are the determination of the parameter region in which dynamical stabilisation occurs, and of the parameter dependence of the width of the stabilised region. For $k<k_{\min }$ the invasion consists of a simple transition wave. When $k_{\text {max }}<k_{H B}$, dynamical stabilisation occurs for $k \in\left(k_{\min }, k_{\max }\right)$; for $k$ just above $k_{\max }$ there are spatiotemporal oscillations immediately behind the invasion front, in the opposite direction. When $k_{\max }>k_{H B}$, dynamical stabilisation occurs for most of the interval $k_{\min }<k<k_{H B}$; it is lost for $k$ just below $k_{H B}$ because of the slow decay behind the invasion front, as discussed in Sect. 4. For $k$ just above $k_{H B}$ there are spatiotemporal oscillations immediately behind the invasion that move with the same speed and direction as the invasion. Our results indicate that there is a critical value of $b \in(1.2,3.0)$ at which there is switch between these behaviours. We calculated this critical value by first computing $k_{H B}$ and $c_{H B}\left(k_{H B}\right)=c_{i n v}\left(k_{H B}\right)$ for a range of $b$ values; this can be done by numerical continuation of the travelling wave equations. For this grid of $b$ values, we then calculated $V^{*}\left(k_{H B}\right)$ using the methods described in Sect. 3; recall that $\left(-V^{*}, V^{*}\right)$ is the range of velocities for which the coexistence steady state has growing linear modes. Now $k_{\text {max }}$ is defined by $V^{*}=c_{i n v}$. Therefore $k_{\max }>k_{H B} \Leftrightarrow V^{*}\left(k_{H B}\right)<c_{H B}\left(k_{H B}\right)$. Figure 12a shows a plot of $V^{*}\left(k_{H B}\right)$ and $c_{H B}\left(k_{H B}\right)$ against $b$; in Fig. 12b, we see how the upper limit of the extent of dynamical stabilisation varies with $b$ by plotting $k_{H B}$ and $k_{\text {max }}$ as functions of $b$. The curves $c_{H B}\left(k_{H B}\right)$ and $V^{*}\left(k_{H B}\right)$ in Fig. 12a, and $k_{H B}$ and $k_{\text {max }}$ in Fig. 12b, intersect at the critical value of $b=1.758$.

Other values of $b$ do not give any behaviour that differs qualitatively from that described for $b=1.2$ and $b=3.0$, and the methods that we have presented can be used to determine the occurrence and extent of dynamical stabilisation. As an example, Fig. 13 illustrates the variation in the width coefficient $\mathcal{W}$, which is proportional to the width of the stabilised region, for $1.2<b<2.1$ and $7.8<k<8$.7. Dynamical stabilisation occurs throughout this parameter region, with no point-to-periodic waves, and the width of the stabilised region is a decreasing function of both $b$ and $k$.
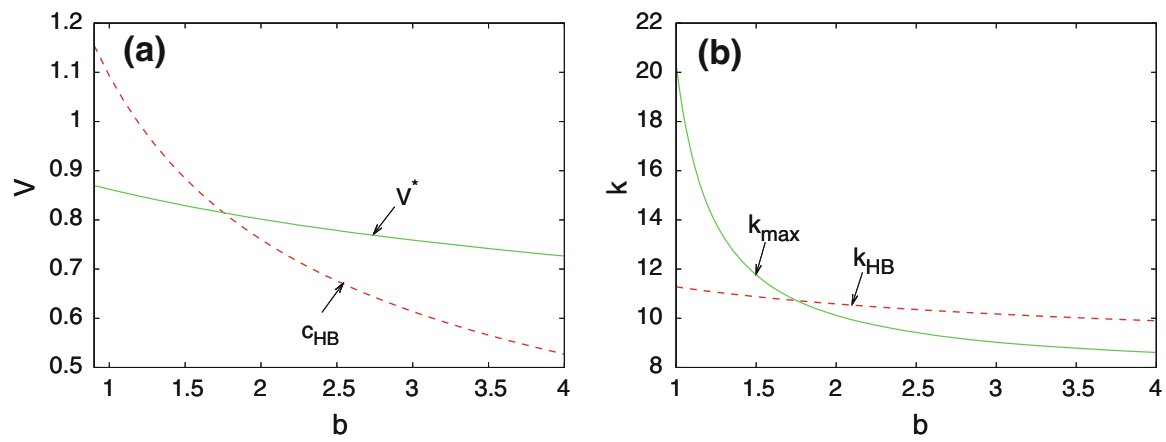

Fig. 12 a Shows plots of $c_{H B}\left(k_{H B}\right)$ (dotted red) and $V^{*}\left(k_{H B}\right)$ (solid green) against $b$. The two curves intersect at 1.758 as do the curves $k_{H B}$ (dotted red) and $k_{\max }$ (solid green) shown on (b). For values of $b$ below this, $k_{H B}<k_{\max }$ implying a point-to-periodic cycle invasion front. However, $k_{H B}>k_{\max }$ for $b>1.758$, meaning that there is a heteroclinic connection behind the invasion front (colour figure online) 

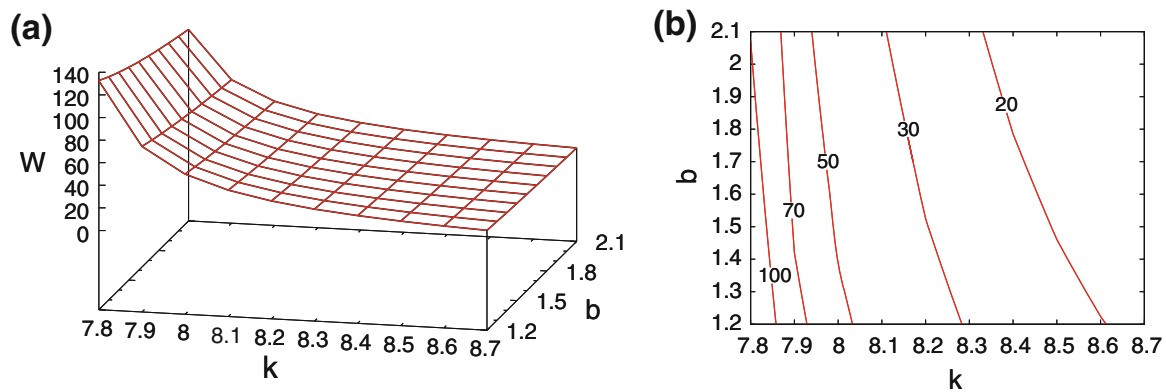

Fig. 13 On panel (a), we show a three-dimensional plot of the width coefficient $\mathcal{W}$ as a function of $b$ and $k$. Featured on panel (b) are contour plots of $\mathcal{W}$ at levels 20, 30, 50, 70 and 100 in the $k, b$ plane. The plots are based on calculations of $\mathcal{W}$, using the method described in the main text, for $1.2<b<2.1$ and $7.8<k<8.7$

\section{Application to Vole-Weasel interactions}

As an example application of our approach to a specific ecological system, we consider the invasion of Microtus voles by their specialist predator the least weasel (Mustela nivalis). Multi-year cycles in the abundence of these voles is a classic and much-studied aspect of the ecology of northern Fennoscandia. The cause of these cycles has been the subject of extensive debate, but a significant volume of data from predator exclusion experiments now points to predation as a leading cause of vole cycling (Korpimäki and Norrdahl 1998; Korpimäki et al. 2002). However it should be noted that vole cycles in other locations, such as Northern UK, appear to result from different mechanisms (Graham and Lambin 2002; Oli 2003; Reynolds et al. 2012).

Parameterisation of the equations $(1 \mathrm{a}-\mathrm{d})$ for the case of the vole-weasel interaction in Northern Fennoscandia is made possible by the large volume of relevant field and laboratory data. The reproductive rate of Microtus voles is relatively uniform across different locations in Northern Europe. We take the maximum per capita birth rate to be 3.3 year $^{-1}$, following Sherratt et al. (2002). This is based on an average litter size of 5, with six litters per season in optimal conditions, and with early-born females breeding themselves in the same season (Dyczkowski and Yalden 1998; Norrdahl and Korpimäki 2002). However it should be noted that other authors have proposed higher estimates (Turchin and Hanski 1997).

For weasels, the corresponding numbers are a mean litter size of 6 , with two litters per season when prey is abundent, and with the females from the first litter breeding themselves in the same season (King 1989; McDonald and Harris 2002). This implies a maximum annual productivity of 30 per adult female weasel, and hence a maximum per capita productivity of 15 year $^{-1}$. The corresponding per capita birth rate is $\log _{e} 15=2.7$ year $^{-1}$. Annual mortality for weasels is $77.5 \%$ (King 1989), implying a mean annual death rate of $-\log _{e} 0.225=1.5$ year $^{-1}$. Using standard nondimensionalisation rescalings for the Rosenzweig-MacArthur model (e.g.Sherratt et al. 2002), these estimates imply $a=1.8$ and $b=1.2$. Given these values, the dimensionless parameter $k$ can be estimated based on the amplitude of the vole population cycles. For $a=1.8$ and $b=1.2$, the 

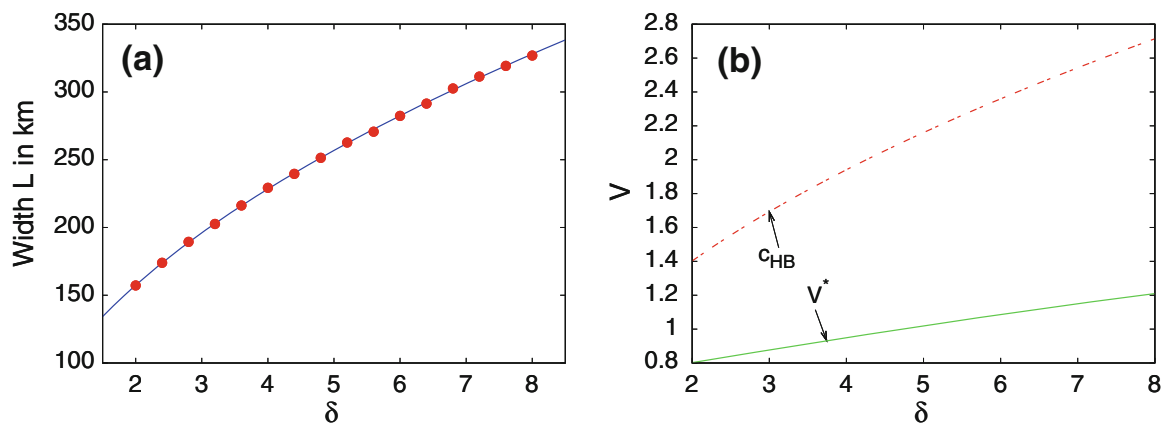

Fig. 14 Panel (a) plots (in red dots) the width $\mathcal{L}$ (in $\mathrm{km}$ ) of the stabilised region against the dispersal ratio $\delta$ with the other ecological parameters fixed as stated in Sect. 6. For each $\delta$ in the range $(2,8)$, we calculated $\mathcal{L}$ in numerical simulations of 1 by averaging the widths measured on PDE simulations on the domain $(0$, 8000) at times between 1000 and 3000 as described in Sect. 3; according to Sherratt et al. (2002), this time range corresponds to a period of 303-909 years after the beginning of invasion. The dimensionless width obtained is then converted into kilometers through multiplication by a factor of 0.246 (see Sherratt et al. 2002). We also calculated the coefficient $\mathcal{W}$ as function of $\delta$ then fitting the converted width $\mathcal{L}$ with $\mathcal{W}$ through linear regression, we plotted the obtained expression of the width in terms of $\mathcal{W}$ as a function of $\delta$ (blue curve). Panel (b) shows $c_{H B}$ and $V^{*}$ as functions of $\delta$. This indicates that for $\delta$ in the range considered, when $k$ rises above $k_{H B}(\delta)$, point-to-periodic invasions occur and there is no stabilised region; for all $\delta \in(2,8), k_{H B}(\delta)$ is bigger than 4.5 , our chosen $k$ value for vole-weasel simulations (colour figure online)

kinetics of (1a-d) have a Hopf bifurcation at $k=3.5$, and the cycle amplitude increases monotonically with $k$ above this value. Field data shows that vole cycle amplitude varies significantly between locations in Northern Fennoscandia (Turchin 2003, §12.2), and we take $k=4.5$; this implies a ratio of maximum to minimum vole densities of about 21 , which is a reasonable representative value.

In comparison with the large volume of data on demographic parameters, there is very limited information on vole dispersal. The mark-capture data of Sherratt et al. (2002) suggests $0.2 \mathrm{~km}^{2}$ year $^{-1}$ as a reasonable estimate of the vole diffusion coefficient. For weasels we are not aware of any relevant data, although the diffusion coefficient will certainly be larger than that for voles. Therefore we varied the dispersal ratio $\delta(>1)$ with $a=1.8, b=1.2$ and $k=4.5$ fixed. Figure $14 \mathrm{a}$ shows that the width of the stabilised region is an increasing function of $\delta$, and that even for the relatively modest estimate $\delta=2$, the width is significant in comparison to the length scale of typical vole habitats in Northern Europe (10's of km). This implies that the dynamical stabilisation phenomenon would be of major significance in the aftermath of an invasion of Microtus voles by least weasels. Figure $15 \mathrm{a}-\mathrm{c}$ show simulations of invasions for $\delta=2,5$ and 8 , which confirm these trends.

Dynamical stabilisation occurs for all of the values of $\delta$ considered in Figs. 14a and $15 \mathrm{a}-\mathrm{c}$; in particular there are no point-to-periodic waves. This is confirmed in Fig. $14 \mathrm{~b}$, which shows that $c_{H B}$ is greater than $V^{*}$ throughout this parameter region. However, this result depends on the value of $k$, which is expected to vary between geographical locations in view of the observed variations in cycle amplitude. To illustrate this, we show in Fig. 15d a simulated invasion for $k=5$, with other parameters 

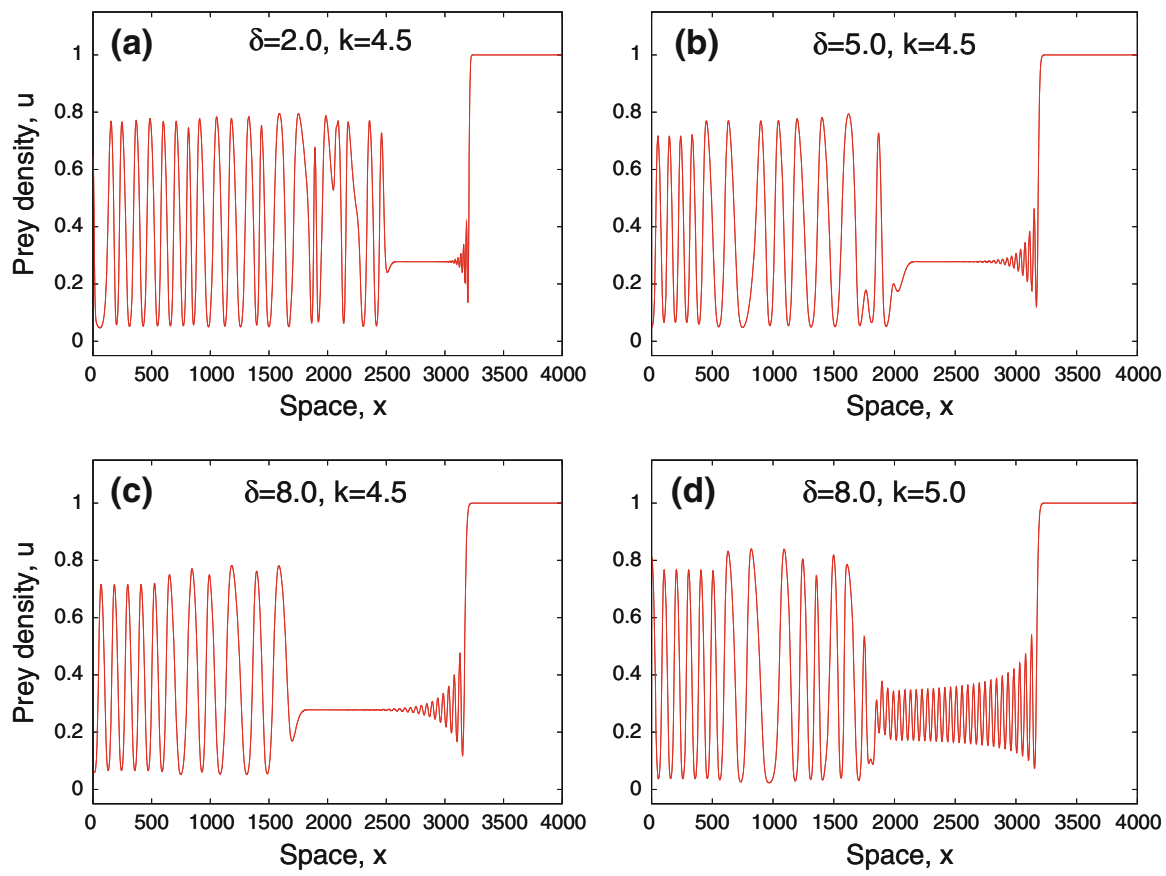

Fig. 15 PDE simulations for various values of $\delta$. For panels (a)-(c), the parameter $k$ is fixed at 4.5 while $\delta$ is respectively 2, 5 and 8 . These pictures show that the width of the stabilised region grows with the dispersal ratio $\delta$, as predicted by Fig. 14a. Panel (d) corresponds to a simulation for $\delta=8$ with $k=5$ which is slightly above the upper limit of the extent of stabilisation $\left(k_{H B}=4.945\right)$; the slow decay seen on panel (c) behind the invasion front has now given way to oscillations of relatively bigger amplitude. Initial conditions, boundary conditions and the numerical method are the same as in Fig. 2

as in Fig. 15c. In this case there is a point-to-periodic invasion, and thus no dynamical stabilisation.

\section{Future mathematical challenges}

We have focussed on the existence and extent of the coexistence steady state as a spatiotemporal transient. The invasion of a prey population by predators can also involve other spatiotemporal transients. In many cases, periodic travelling waves develop, moving in the opposite direction to the invasion, and in some cases these appear in a moving band (Fig. 2c, d). Additionally the periodic travelling waves moving with the invasion for $k>k_{H B}$ typically occur in a spatiotemporally transient band (Figs. 9d, $15 \mathrm{~d})$. We expect that both of these phenomena could be investigated using the same methodology as in this paper. However, there is one very fundamental practical difficulty: there is currently no systematic algorithm for computing the absolute spectrum for a non-constant solution of a partial differential equation. There is no particular difficulty in performing a numerical continuation of the absolute spectrum; the problem lies in the identification of appropriate starting parts (Rademacher et al. 2007). Our work highlights the importance of research on the calculation of the absolute spectrum for non-constant solutions. 


\section{References}

Dunbar SR (1984) Travelling wave solutions of diffusive Lotka-Volterra equations: a heteroclinic connection in $\mathrm{R}^{4}$. Trans Am Math Soc 268:557-594

Dunbar SR (1986) Travelling waves in diffusive predator-prey equations: periodic orbits and point-toperiodic heteroclinic orbits. SIAM J Appl Math 46:1057-1078

Dyczkowski J, Yalden DW (1998) An estimate of the impact of predators on the British field vole Microtus agrestis population. Mammal Rev 28:165-184

Fraile JM, Sabina JC (1989) General conditions for the existence of a critical point-periodic wave front connection for reaction-diffusion systems. Nonlinear Anal Theory Methods Appl 13:767-786

Garvie MR (2007) Finite difference schemes for reaction-diffusion equations modeling predator-prey interactions in MATLAB. Bull Math Biol 69:931-956

Graham IM, Lambin X (2002) The impact of weasel predation on cyclic field-vole survival: the specialist predator hypothesis contradicted. J Anim Ecol 71:946-956

Hellmann JJ, Byers JE, Bierwagen BG, Dukes JS (2008) Five Potential consequences of climate change for invasive species. Cons Biol 22:534-543

King CM (1989) The natural history of weasels and stoats. Christoper Helm, London

Kopell N, Howard LN (1973) Plane wave solutions to reaction-diffusion equations. Stud Appl Math 52:291328

Korpimäki E, Norrdahl K (1998) Experimental reduction of predators reverses the crash phase of smallrodent cycles. Ecology 79:2448-2455

Korpimäki E, Norrdahl K, Klemola T, Pettersen T, Stenseth NC (2002) Dynamic effects of predators on cyclic voles: field experimentation and model extrapolation. Proc R Soc Lond B 269:991-997

Lotka AJ (1925) Elements of physical biology. Williams and Wilkins, Baltimore

Malchow H, Petrovskii SV (2002) Dynamical stabilization of an unstable equilibrium in chemical and biological systems. Math Comput Model 36:307-319

Malchow H, Petrovskii SV, Venturino E (2008) Spatiotemporal patterns in ecology and epidemiology. Chapman and Hall/CRC, Boca Raton

Matsumura C, Yokoyama Y, Washitani I (2004) Invasion status and potential ecological impacts of an invasive alien bumblebee, Bombus Terrestris L. (Hymenoptera: Apidae) naturalized in Southern Hokkaido. Jpn Glob Environ Res 8:51-66

May RM, Mclean AR (2007) Theoretical ecology: principles and applications. Oxford University Press, Oxford

McDonald RA, Harris S (2002) Population biology of stoats Mustela erminea and weasels Mustela nivalis on game estates in Great Britain. J Appl Ecol 39(5):793-805

Norrdahl K, Korpimäki E (2002) Changes in population structure and reproduction during a 3-yr population cycle of voles. OIKOS 96(2):331-345

Nozaki K, Bekki N (1983) Pattern selection and spatiotemporal transition to Chaos in the Ginzburg-Landau equation. Phys Rev Lett 51:2171-2174

Oli MK (2003) Population cycles of small rodents are caused by specialist predators: or are they? Trends Ecol Evol 18:105-107

Owen MR, Lewis MA (2001) How predation can slow, stop or reverse a prey invasion. Bull Math Biol 63:655-684

Petrovskii SV, Vinogradov ME, Morozov AY (1998) Spatial-temporal dynamics of a localized populational "burst" in a distributed prey-predator system. Okeanologiya 38:881-890

Petrovskii SV, Vinogradov ME, Morozov AY (2000) Spatial-temporal dynamics of a localized populational "burst" in a distributed prey-predator system. Okeanologiya 38:37-51

Petrovskii S, Kawasaki K, Takasu F, Shigesada N (2001) Diffusive waves, dynamical stabilization and spatio-temporal chaos in a community of three competitive species. Jpn J Indust Appl Math 18:459-481

Rademacher JDM, Sandstede B, Scheel A (2007) Computing absolute and essential spectra using continuation. Phys D 229:166-183

Reynolds JJH, Massey FP, Lambin X, Reidinger S, Sherratt JA, Smith MJ, White A, Hartley SE (2012) Delayed induced silica defences in grasses and their potential for destabilising herbivore population dynamics. Oecologia 170:445-456

Rosenzweig ML, Macarthur RH (1963) Graphical representation and stability conditions of predator-prey interactions. Am Natur 97:209-223 
Sandstede B, Scheel A (2000) Absolute and convective instabilities of waves on unbounded and large bounded domains. Phys D 145:233-277

Sherratt JA (2001) Periodic travelling waves in cyclic predator-prey systems. Ecol Lett 4:30-37

Sherratt JA, Smith MJ (2008) Periodic travelling waves in cyclic populations: field studies and reactiondiffusion models. J R Soc Interface 5:483-505

Sherratt JA, Eagan BT, Lewis MA (1997) Oscillations and chaos behind predator-prey invasion: mathematical artifact or ecological reality. Philos Trans R Soc Lond B 352:21-38

Sherratt TN, Lambin X, Petty SJ, MacKinnon JL, Coles CF, Thomas CJ (2000) Application of coupled oscillator models to understand extensive synchrony domains and travelling waves in populations of the field vole in Kielder forest, UK. J Appl Ecol 37(suppl. 1):148-158

Sherratt JA, Lambin X, Thomas CJ, Sherratt TN (2002) Generation of periodic waves by landscape features in cyclic predator-prey systems. Proc R Soc Lond B 269:327-334

Sherratt JA, Smith MJ, Rademacher JDM (2009) Locating the transition from periodic oscillations to spatiotemporal chaos in the wake of invasion. PNAS 106:10890-10895

Smith MJ, Sherratt JA (2009) Propagating fronts in the complex Ginzburg-Landau equation generate fixedwidth bands of plane waves. Phys Rev E 80 (Art. No. 046209)

Smith MJ, Rademacher JDM, Sherratt JA (2009) Absolute stability of wavetrains can explain spatiotemporal dynamics in reaction-diffusion systems of Lambda-Omega type. SIAM J Appl Dyn Syst 8:1136-1159

Tokarska-Guzik B, Brock JH, Brundu G, Child L, Daehler CC, Pysek P (2008) Plant invasions: human perception, ecological impacts and management. Backhuys, Leiden

Turchin P (2003) Complex population dynamics: a theoretical/empirical synthesis. Princeton University Press, Princeton

Turchin P, Hanski I (1997) An empirically based model for lattitudinal gradient in vole population dynamics. Am Nat 149:842-874

van Saarloos W (2003) Front propagation into unstable states. Phys Rep 386:29-222

Volterra V (1926) Variazione fluttuazioni del numero d'individui in specie animali conviventi. Mem Acad Lincei 2:31-113 (Translation in an appendix to Chapman RN (1931) J Anim Ecol 409-448) 